\title{
Epidemiología de las intoxicaciones en Chile: una década de registros
}

\author{
Cristián Mena $\mathbf{H}^{\mathrm{a}}$, Marli Bettini $\mathbf{S}^{\mathbf{b}}$, Patricia Cerda J ${ }^{\mathbf{b}}$, \\ Flor Concha $\mathrm{S}^{\mathrm{b}}$, Enrique Paris $\mathrm{M}$. \\ Epidemiology of intoxications in \\ Chile: ten years of registry
}

Background: The existence of Poison Centers for management and prevention of intoxications has been endorsed by the international experience. In Chile, the Toxicological Information Center at the Pontifical Catholic University of Chile has been active since 1992, receiving about 130.000 calls until 2002. Aim: To analyze the statistical data gathered throughout the first ten years of our Research Center. To delineate the epidemiological pattern of intoxications in Chile. Material and methods: Retrospective study in which records from calls for toxicological information received during the 1992-2002 period reviewed. Analyzed data were total calls per year, place of call, exposure circumstances, age, sex, route of exposure and involved agents. Results: 96,468 calls analyzed. The main exposure circumstance was «unintentional»(78.6\%), followed by «ntentional»(16.9\%). Intoxications in children under 5 years old motivated $50 \%$ of calls. According to route of exposure, ingestions involved 75,992 calls (78.8\%). Medications were the most common substances, accounting for $49.2 \%$ of calls, followed by cleaning products (12.1\%), pesticides (11.3\%), industrial and chemical products $(10.5 \%)$ and cosmetics (2.7\%). Medications acting on the CNS were the most recurrent, with 19,096 reports. Conclusions: The epidemiological pattern for intoxications in Chile is very similar to that reported in developed and other Latin American countries. Children under 5 years old, are a high risk group for intoxications. It is imperative to improve the recording and followup of patients that call to the Center, to improve epidemiological data of intoxications in Chile (Rev Méd Chile 2004; 132: 493-9).

(Key Words: Poisoning; Poison Control Centers; Toxicology)

Recibido el 29 de julio, 2003. Aceptado en versión corregida el 24 de septiembre, 2003.

Centro de Información Toxicológica y de Medicamentos, Facultad de Medicina de la Pontificia Universidad Católica de Chile.

anterno de Medicina, Facultad de Medicina, Pontificia Universidad Católica de Chile

benfermera-Matrona

Correspondencia a: Cristián Mena Henríquez. CITUC. Marcoleta 367, Casilla 114-D, Santiago, Chile. Fono: 56-26353800. Fax: 56-2-2472112. E mail: cituc@med.puc.cl; cimena@puc.cl 
L a existencia de Centros Toxicológicos para el manejo y la prevención de las intoxicaciones ha sido ampliamente avalada por la experiencia de países desarrollados y por directrices de la Organización Mundial de la Salud (OMS) en 19981. En América Latina existen centros, en diversos países, que otorgan información tanto al personal médico como al público general. Los datos recopilados por algunos de estos centros han sido comunicados ${ }^{2,3}$, observándose características epidemiológicas similares a la de países desarrollados, con algún grado de variabilidad determinada por factores económicos, culturales, geográficos y de la normativa legal de cada país 2 . En Chile, el Centro de Información Toxicológica y de Medicamentos de la Pontificia Universidad Católica (CITUC) fue fundado en septiembre de 1992, siendo el primer Centro Toxicológico del país inserto dentro de una Facultad de Medicina, en prestar servicios los 365 días del año en horario continuo, recibiendo hasta el año 2002 cerca de 130.000 llamadas, provenientes de diversas regiones del país. Durante sus 10 años de funcionamiento, el centro se ha posicionado como referencia en el manejo del paciente intoxicado, teniendo como tarea principal la orientación al personal de salud y a la comunidad en el manejo y prevención de las intoxicaciones.

El registro de los datos de cada exposición es necesario para la toxicovigilancia y planificación de campañas de prevención. En nuestro centro, este registro se realiza en forma rigurosa mediante la ficha elaborada por el Proyecto IPCS INTOX (The International Programme on Chemical Safety) de la OMS4. Anualmente se han confeccionado estadísticas de estos registros, los cuales han sido presentados en forma parcial ${ }^{4}$ (X Congreso Latinoamericano de Toxicología. La Habana, Cuba. 1998).

El siguiente estudio pretende mostrar la estadística recopilada durante los primeros 10 años de funcionamiento del centro, y de esta manera aproximarse al patrón epidemiológico de las intoxicaciones en Chile.

\section{MATERIAL Y MÉTODO}

Se revisaron los registros anuales de las llamadas recibidas por nuestro centro desde su inauguración en septiembre de 1992. Se excluyeron del análisis los 2 primeros años de funcionamiento, por problemas en el registro de la información, analizándose las llamadas por información toxicológica entre los años 1995 y 2002.

Los siguientes datos fueron analizados: número total de llamadas por año, procedencia de la llamada, circunstancias de la exposición, edad, sexo, vía de exposición, agente involucrado y tipo de medicamento involucrado. La penetrancia de las intoxicaciones, definida como el número de casos de intoxicaciones reportados por cada 1.000 individuos de la población atendida ${ }^{5}$, fue calculada para el año 2002.

Los agentes involucrados fueron clasificados en 6 categorías: medicamentos, productos industriales y químicos, productos de aseo, plaguicidas (domésticos e industriales), cosméticos y otros.

\section{RESUlTADOs}

En la Figura 1 se muestra la evolución de las llamadas recibidas en el Centro de Información Toxicológica y de Medicamentos de la Pontificia Universidad Católica de Chile en sus 10 años de funcionamiento. Un total de 129.406 llamadas fueron reportadas, de las cuales 96.468 fueron analizadas y que corresponden a llamadas por información toxicológica del período 1995-2002. Asumiendo el total de la población nacional como población atendida (15.116.435 habitantes) ${ }^{6}$, la penetrancia para el año 2002 fue de 1,34 casos de intoxicaciones reportados por cada 1.000 habitantes, sin embargo al realizar el mismo cálculo para la Región Metropolitana (centro poblacional más grande del país) este valor ascendió a 2,54 casos de intoxicaciones por cada 1.000 habitantes.

El $64,2 \%$ de las consultas provino desde la residencia de los pacientes y 30,8\% de instalaciones médicas, el restante $5 \%$ corresponde a llamadas provenientes desde el lugar de trabajo del interlocutor, instituciones educativas y lugares públicos, entre otros (Figura 2). La principal circunstancia de exposición fue la no intencional con 78,6\%. Dentro de este grupo, las circunstancias accidentales fueron las más frecuentes y la causa más importante de llamadas recibidas por nuestro centro. Las circunstancias intencionales correspondieron a $16,4 \%$, destacando en esta categoría las causas intencionales-suicidas (Figura 3). 


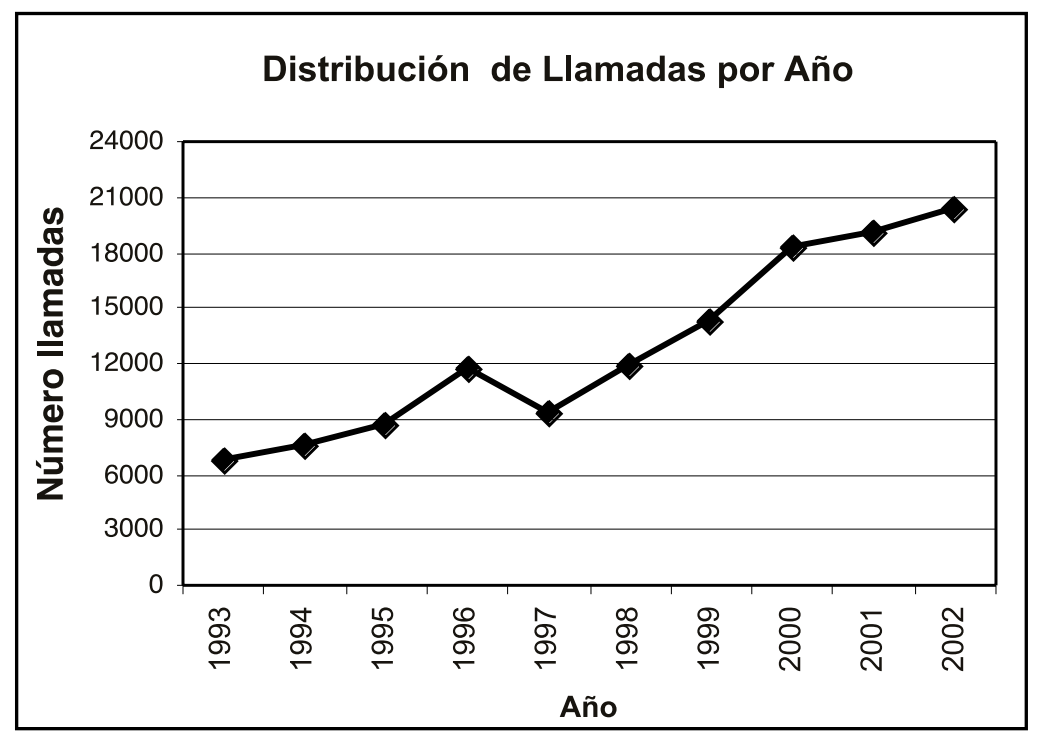

Figura 1. Número de llamadas por año. CITUC, período 19922002.

FIgura 2. Procedencia de llamadas. CITUC, período 1995-2002.

\section{Procedencia de la llamada}

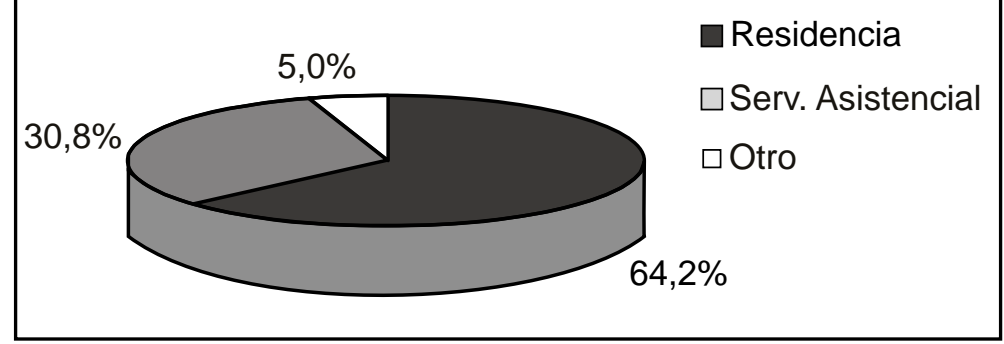

\section{Circunstancia de la Exposición}

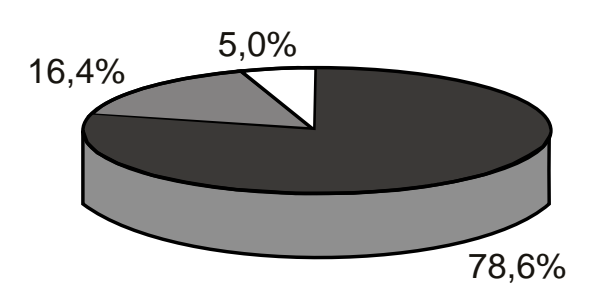

$\square$ No-intencional

$\square$ Intencional

口Otras

FIgURA 3. Circunstancias de la exposición toxicológica. CITUC, período 1995-2002. 
Según grupo de edad (Figura 4), la mayor cantidad de llamadas correspondió a menores de 5 años con 50\% (48.214). En el grupo de 6 a 20 años, las consultas representaron $20,4 \%$ con 19.633 llamadas, el restante $29,7 \%$ fueron pacientes mayores de 20 años.

El 48\% de las llamadas correspondió a pacientes del sexo masculino y 46,3\% a mujeres.

Respecto a la vía de exposición, las ingestiones involucraron 75.992 llamadas $(78,8 \%)$ seguidas por la vía inhalatoria con $6,8 \%$, las picaduras y mordeduras (principalmente por arañas venenosas) con $6 \%$ y la vía dérmica con $3,4 \%$; el $5 \%$ restante corresponde a la vía ocular, parenteral y mucosas (Figura 5).

Los medicamentos fueron las sustancias más comúnmente implicadas en las intoxicaciones, seguidos por los productos de aseo, plaguicidas (domésticos y agrícolas), productos industriales y químicos y productos cosméticos (Figura 6). En la categoría «otros») encontramos los reportes por metales, cuerpos extraños, gases, plantas y alimentos.

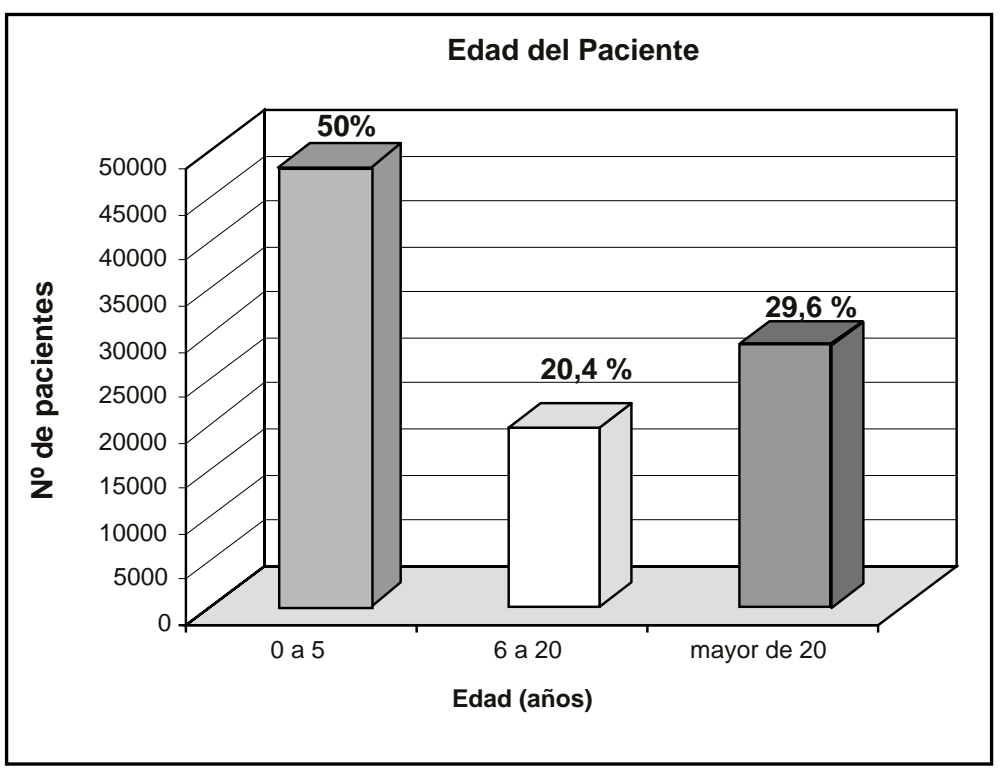

FIgURA 4. Distribución por edad de pacientes expuestos a intoxicaciones. CITUC, período 19952002.

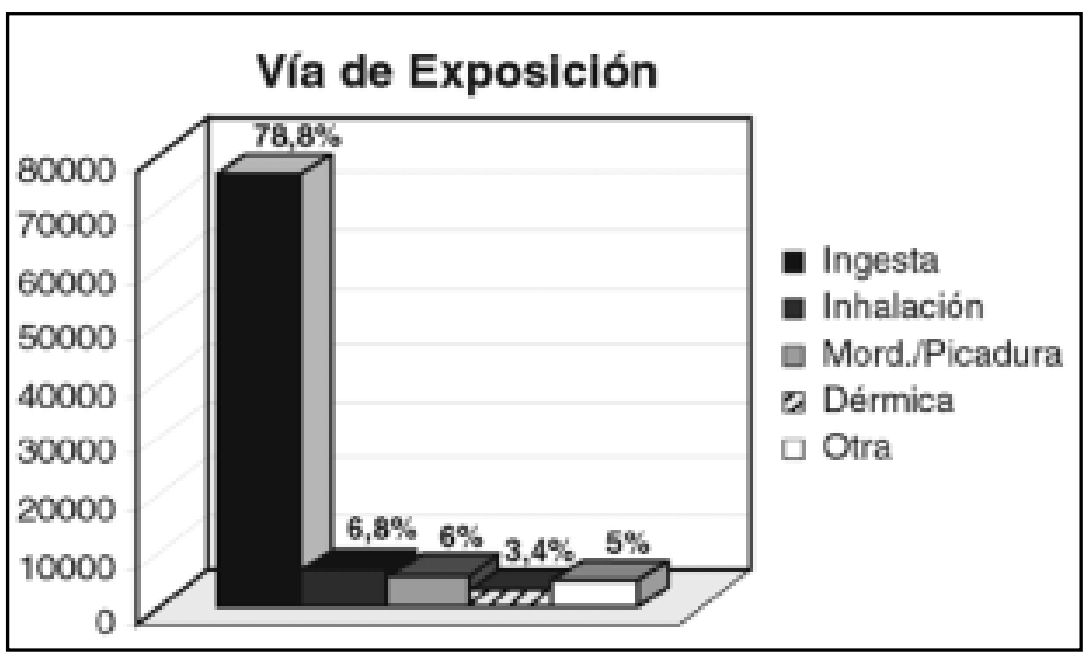

FIgURA 5. Vía de exposición al agente tóxico. CITUC, período 1995-2002. 
Los medicamentos que actúan a nivel de sistema nervioso central fueron los más recurrentes con 19.096 reportes (40,3\%), los cuales incluyeron benzodiacepinas, anticonvulsivantes, antidepresivos (tricíclicos e inhibidores selectivos de la recaptación de serotonina), fenotiazinas y narcóticos-opiáceos entre otros. Le siguen los medicamentos que actúan en el sistema respiratorio -antihistamínicos, broncodilatadores, mucolíticos, antitusígenos- con 10,9\%, antimicrobianos $6,2 \%$, hormonales con $5,5 \%$ y finalmente las drogas que actúan a nivel cardiovascular (antiarrítmicos, antihipertensivos, vasodilatadores) con 4,7\% (Figura 7).

\section{DISCUSIÓN}

$\mathrm{Si}$ bien existen actualmente en Chile varios centros que otorgan orientación toxicológica, éste es el primer estudio que muestra información sobre las intoxicaciones en Chile en un período mayor a un año.

Los resultados aquí presentados corresponden a la estadística recopilada entre los años 1992 y 2002. Jaraczewska et al han descrito previamente los tipos de centros toxicológicos existentes a nivel mundial. A partir de lo anterior, nuestro centro está categorizado como privado, dependiente de un hospital universitario, que funciona

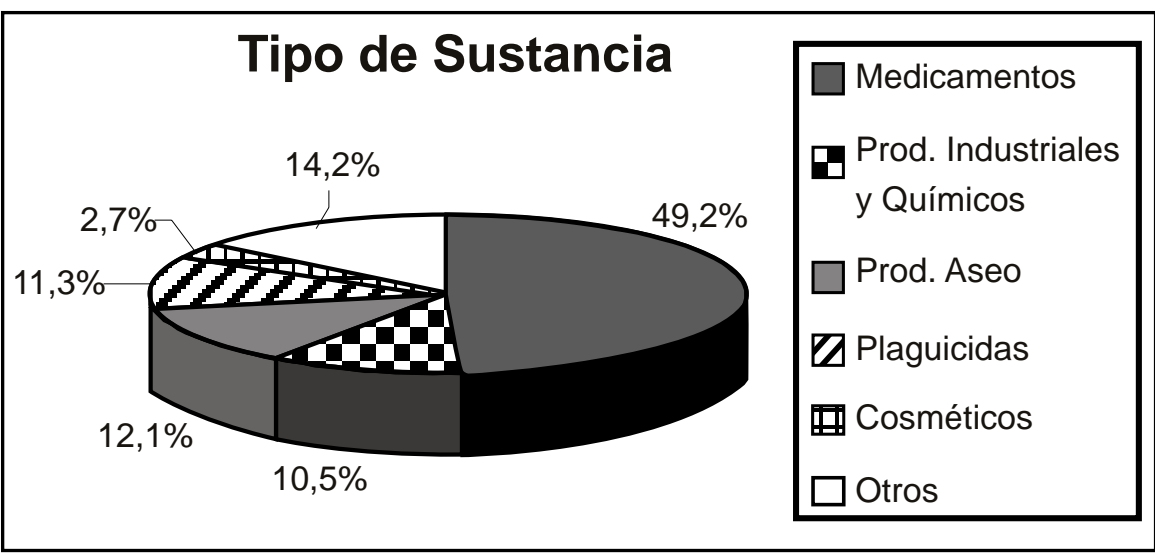

FIguRa 6. Sustancias involucradas en accidentes toxicológicos. CITUC, período 19952002.

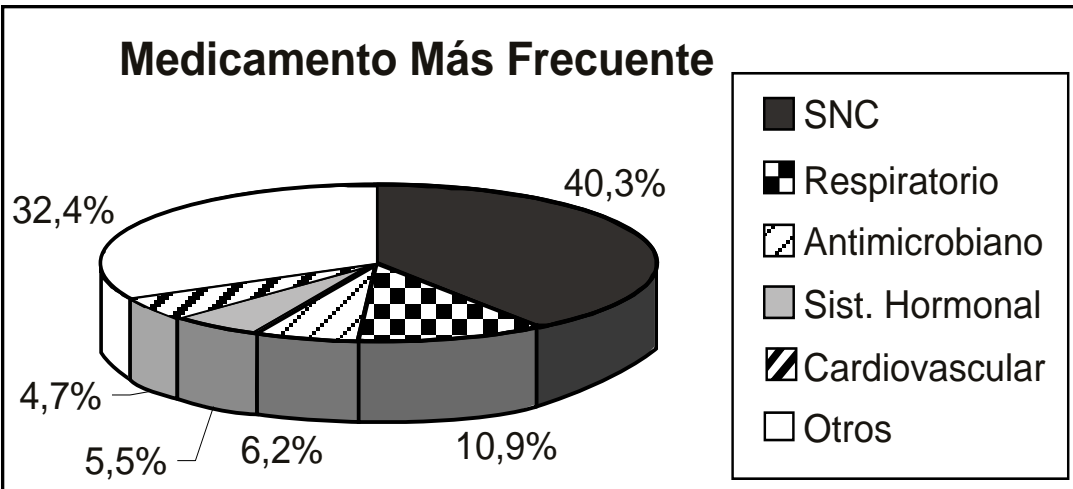

Figura 7. Medicamentos más frecuentes involucrados en intoxicaciones. CITUC, período 1995-2002. 
exclusivamente con atención telefónica, gratuito y que no cuenta con laboratorio propio o camas destinadas a tratar casos de intoxicación ${ }^{7}$.

Durante el período analizado, se aprecia una tendencia ascendente del número de llamadas recibidas, con un crecimiento desde 6.761 en 1993 a 20.313 llamadas en 2002, esta última cifra en el rango de centros toxicológicos de países desarrollados ${ }^{6}$. Creemos que estos resultados se explican mejor por un aumento en la notificación de los casos y no por un incremento en la incidencia de episodios toxicológicos. A pesar de lo anterior, podemos sospechar que el número de incidentes aún está subnotificado, hecho que se refleja en la penetrancia obtenida en el año 2002 para la Región Metropolitana (2,54 casos de intoxicación notificados por cada 1.000 individuos de población atendida), la cual está muy por debajo de las reportadas por centros de países desarrollados (promedio de 8,1 casos notificados/1.000 habitantes para Estados Unidos) ${ }^{5}$.

Los resultados obtenidos con relación a la vía de exposición, procedencia de la llamada, causa de la exposición, distribución por sexo y por edad, son similares a los observados en estudios previos de Argentina ${ }^{3}$, Costa Rica ${ }^{2}$, Alemania ${ }^{8}$ y Estados Unidos ${ }^{5}$. Existe $6 \%$ de exposiciones por picaduras/mordeduras, las cuales estarían relacionadas principalmente con consultas por Loxosceles laeta (araña de rincón).

La mayoría de las llamadas recibidas se concentró en niños menores de 5 años. Los factores que podrían explicar este resultado incluyen el fácil acceso que este grupo etáreo tiene a diversas sustancias tóxicas, el almacenaje inadecuado de

\section{REFERENCIAS}

1. OMS, PNUMA, OIT: Directrices para la lucha contra las intoxicaciones. Programa internacional de seguridad de las sustancias químicas. Ginebra 1998.

2. Leveridge $Y$. The pattern of poisoning Costa Rica during 1997. Vet Hum Toxicol 1999; 41: 100-2.

3. Primer informe estadístico de consultas registradas por los CIAATS (centros de informa- los productos, trasvasije de sustancias a envases atractivos para los menores y ausencia de una legislación que exija envases resistentes a niños para las sustancias potencialmente tóxicas, especialmente medicamentos.

El predominio de los medicamentos como causa de intoxicación coincide con los trabajos ya citados. Dentro de éstos, la mayor frecuencia de fármacos que actúan sobre el sistema nervioso central podría explicarse por la facilidad que existe en Chile para acceder a antidepresivos, antipsicóticos y anticonvulsivantes, pues la legislación actual sólo regula la compra, bajo receta médica retenida, de benzodiacepinas y opioides (en este grupo farmacológico). Los productos de aseo ocupan el segundo lugar en frecuencia. Reportes previos señalan que la mayor frecuencia de exposición de una determinada sustancia no está necesariamente en relación con su potencial tóxico ${ }^{9}$, sin embargo, se necesita de un mayor reporte de datos para poder extrapolar esta información a nuestro país.

Los datos aquí presentados son una aproximación a la epidemiología de las intoxicaciones en Chile. Creemos que su fortaleza está en el volumen de las llamadas analizadas (96.468) y la rigurosidad en su registro, los cuales permiten dar validez y peso estadístico a los resultados mostrados. Sin embargo, no ha sido posible acceder a otros datos epidemiológicos importantes como mortalidad, evolución clínica y tratamientos recibidos por los pacientes.

Finalmente, es necesario mejorar el seguimiento de los pacientes que consultan en el centro, para poder así completar esta visión inicial de la epidemiología de las intoxicaciones en Chile.

ción, asesoramiento y asistencia toxicológica) de la República Argentina año 2000. Ministerio de Salud de la Nación, Argentina. 2000.

4. París E, Ríos JC. Intoxicaciones: Epidemiología, clínica y tratamiento. Ediciones Universidad Católica de Chile. Santiago, Chile. 2001; 13-27.

5. Lutovitz T, Kiein-Schwartz W, Rodgers G, Cobaugh D, Youniss J, OMSLAER J et al. 2001 annual reports of the American Association of poison control centers toxic exposure surveillance system. Am J 
Emerg Med 2002; 20: 391-452.

6. Instituto Nacional de Estadísticas. Censo 2002. Síntesis de Resultados. Instituto Nacional de Estadísticas. Chile. 2003.

7. Jaraczewska W, Czerczak S, Kortwica M. The National Poison Infomation Center in Poland. J Toxicol Clin Toxicol 1996; 34: 669-72.

8. Wolfle J, Kowalewski S. Epidemiology of ingestio- ns in a regional poison center over twenty years. Vet Hum Toxicol 1995; 37: 367-8.

9. LUTOVItz T, ManoguerRa A. Comparison of pediatric poisoning hazards: an analysis of 3.8 million exposure incidents. A report from the American Association of Poison Control Centers. Pediatrics 1992; 89: 999-1006. 